\title{
Perineal Myxoid Liposarcoma - Computed Tomography and Magnetic Resonance Imaging Findings: A Case Report
}

\author{
Hadi Sasania, c , Baris Bakira, Rustu Turkay ${ }^{\mathrm{a}}$, Memduh Dursun ${ }^{\mathrm{a}}$, Suleyman Bademler ${ }^{\mathrm{b}}$
}

\begin{abstract}
Myxoid liposarcoma (MLS) is a major subtype of liposarcomas accounts for $30-50 \%$ of all the liposarcomas. Perineal located MLS is rare. Due to the tumor content it may have a variable spectrum of imaging abnormality in cross-sectional imaging modalities. In this article, Computed Tomography and Magnetic resonance Imaging findings of a MLS case in the perineal region with the review of the literature will be presented.
\end{abstract}

Keywords: Perineal myxoid liposarcoma; Computed tomography; Magnetic resonans imaging

\section{Introduction}

Liposarcomas are rare soft tissue tumors, derived from primitive mesenchymal cells [1]. Myxoid liposarcoma (MLS) is a major subtype of this group, consisting about 30$50 \%$ of all the liposarcomas, presenting between second and seven decade of life (between 18 - 67 years with a mean age of 42 years) [2]. Their usual location sites are extremities specially in the thigh of the lower extremity. Perineum and pararectal spaces are rare, intrascrotal is very rare location sites of MLS [1, 2-4] MLS has variable appearance spectrum on cross-sectional imaging modalities due to its fatty and matrix content $[5,6]$.

In this article, Computed Tomography and Magnetic Resonance Imaging findings of a rare seen perineal located

\footnotetext{
Manuscript accepted for publication September 24, 2012

${ }^{a}$ Departments of Radiology, Istanbul University, Istanbul Medical, Faculty, 34093, Istanbul, Turkey

${ }^{\mathrm{b}}$ General Surgery, Istanbul University, Istanbul Medical, Faculty, 34093, Istanbul, Turkey

${ }^{\mathrm{c}}$ Correspondence author: Hadi Sasani, Radiology Specialist. Istanbul University, Medical Faculty of Istanbul University, Department of Radiology, 34093, Istanbul, Turkey. Email: sasani@istanbul.edu.tr

doi: http://dx.doi.org/10.4021/jmc860w
}

Myxoid liposarcoma with the review of the literature, are presented.

\section{Case Report}

A 36 years old male patient with the complaint of pain and swelling at the right perineal region for 3 month duration admitted to Istanbul University Istanbul Medical Faculty Hospital. Althought the swelling was persisting before, it became more prominent during few months. On physical examination at the right perineal region, the located pain and swelling which extended toward the penil root, was detected. On Abdominal Computerized Tomography (CT) demonstrated prominent hypodense area (in fluid density) in the perineal region which was at the location of the right corpus spongiosum, between the both corpus cavernosa and at the right portion of the penil root (Fig. 1).

Pelvic Magnetic Resonans Imaging (MRI) study showed a $8 \times 6 \times 3 \mathrm{~cm}$ sized lesion cystic in nature, hyperintense on T1-Weighted images, remarkable hyperintense in signal intensity on STIR sequence, mural enhancing and heterogenous contrast enhacement pattern on post-contrast imaging was visualized. No relation between the lesion and anal channel was detected (Fig. 2). The lesion was totally removed surgerically. The histopathology was reported as myxoid liposarcoma.

\section{Discussion}

Liposarcoma is the second most common seen soft-tissue sarcoma in adults which is about $14-18 \%$ of all soft-tissue sarcomas $[7,8]$. They originate from primitive mesenchymal cells rather than from mature fat cells [1]. They can be seen everywhere in the body containg the fat tissue. Myxoid liposarcoma (MLS) is a major subtype of these liposarcomas which represents approximately $30-50 \%$ of all the liposarcomas and the mean age is 42 years (between 18 - 67 years ) [2]. MLS commonly presents in the extremities, particularly in the thigh, however, it is relatively rare in the perineum or pararectal spaces $[2,3]$.The major factors in determining 


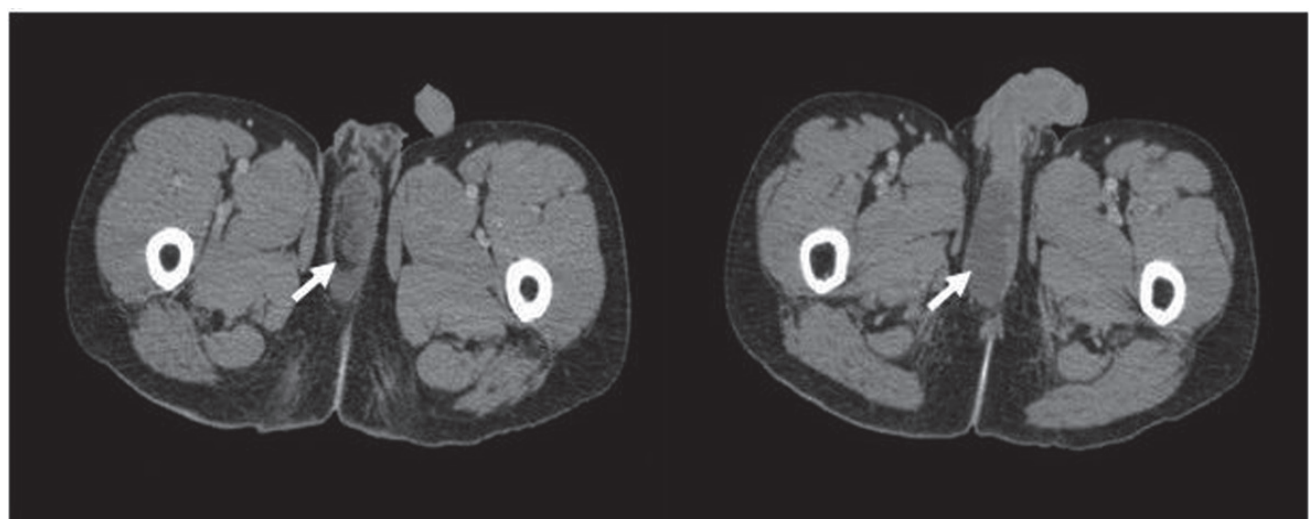

Figure 1. Contrast- enhanced CT of a 36 years old male patient with the complaint of pain and swelling at the right perineal region showed hypodense area in the perineal region (in fluid density) which was at the location of the right corpus spongiosum, between the both corpus cavernosa and at the right portion of the penil root (arrow). (Toshiba Aquilion 64 Detector CT, Istanbul/Turkey. CT Protocol: 50 mAs, 120 kV, 7 mm Slice thickness, $350 \mathrm{~mL}$ iodine contrast was used).

the behavior and prognosis of liposarcoma are the histologic features, site, and size of the tumor $[1,7,9]$. Also the very rare form of MLS involvement in intrascrotal location has been reported [4]. MLS involves the intermuscular fascial planes or deep-seated areas, rarely found in the subcutaneous tissue [5]. Clinically they tend to present as painless swellings that progressively increase in size. MLS usually grow to a considerable size before become clinically symptomatic. The tumor is relatively soft and non-tender at physical examination.

Diagnosis is usually depends on the clinical symptoms (swelling, maybe pain) and the mostly on the imaging. Generally the multi-imaging modalities help for the exact preoperative assessment, diagnosis, staging of MLS, providing the curative resection and minimizing the local recurrence risk in patients. CT and MRI are useful crosssectional imaging modalities, which have large spectrum of signal intensity in MRI and CT attenuation, may provide information about the size, biological behavior, tissue characterization, the tumor extention.

The imaging abnormalities in both modalities (CT and specially for MRI) depends on the amount of fat content (less than $10-25 \%$ of the tumour volume), the degree of cellularity and vascularity, and the presence of necrosis within the tumor, the quantity of mucinous and myxoid materials in the tumor, they may show hyperintense foci (represents fat within the tumor ) in a predominantly homogeneous hypointense mass on T1-weighted images, in intermediate signal intensity On T2-Weighted images $[5,6]$.

The most majority part of MLS contain lacy or linear, amorphous foci of fat. Typically MLS tend to be hypointense on nonenhanced T1-weighted images and hyperintense on T2-weighted images. Contrarily to this, due to their relatively high fat content, lipomas and well-differentiated liposarcomas show high signal intensity on T1-weighted im- ages [5]. Although they may appear as a cystic lesion on nonenhanced MR imaging or they may have similar appearance to a fluid-filled cyst at T2-weighted imaging, some MLS tend to enhance the contrast material like other solid tumors at contrast-enhanced MR study [5]. On Contrast-enhanced images there may be very little non-remarkable contrast enhacement, because of this reason they may mimic a cystic lesion $[2,5]$.

Because of this reason, contrast (Godalinium) enhanced MRI is prefered and essential to differantiate the cystic or necrotic lesions from solid or cellular lesions $[2,3]$ There are three contrast enhancing pattern in MRI including homogeneous (total enhancement), heterogeneous (partial enhancement, intensity more than $80 \%$ of the tumor volume) and no enhancement patterns [5].

The signal intensity of the lesion is variable due to its contents. If any lesion contains an extracellular matrix with a high level of mucopolysaccharide, hyaline cartilage, edema and areas of necrosis tends to be cystic in nature and it appears as a cystic mass on nonenhanced images [10]. It makes the diagnosis difficult to differantiate cystic lesion from solid masses. In this level ultrasonography (US) may provide information about the internal nature of the lesions. If the MLS is purely cystic appearance at MRI, both US and contrastenhanced MR imaging and US are helpful in the differential diagnosis.

MLS may mimic a fluid-filled cyst at T2-weighted imaging, therefore, contrast-enhanced MR imaging is important in differantiating cystic or necrotic lesions from solid, cellular lesions [11, 12].

The local recurrence rates of MLS are relatively high at about $40-50 \%$ [13]. Pure MLS has a rate of metastasis about $20 \%$ to the common sites including lungs, bones, and rarely the serosal surfaces (of the pleura, peritoneum and pericardium) $[2,3]$. It is of great value to mention that the major predicting 


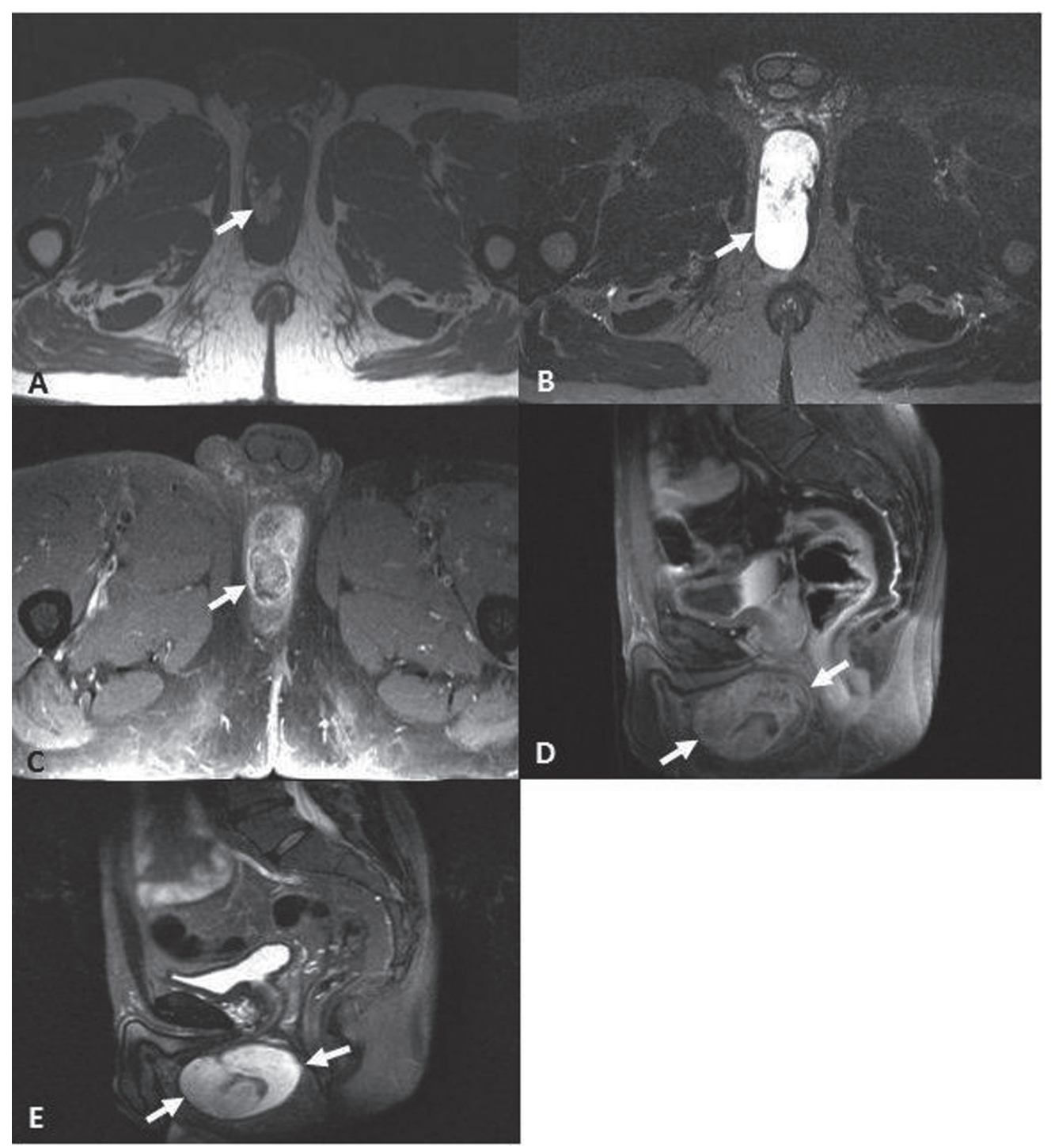

Figure 2. Pelvic MR Imaging of of a 36 years old male patient with the complaint of pain and swelling at the right perineal region revealed a $8 \times 6 \times 3 \mathrm{~cm}$ sized lesion cystic in nature, hyperintense on axial T1-Weighted images (TE: 600, TR: 10) (A), remarkable hyperintense in signal intensity on axial and sagittal STIR sequence TE: 85, TR: 3000) $(B, C)$, heterogenous and mural contrast enhacement pattern on axial and sagittal post-contrast imaging (TE: 635 TR: 15 ), (D, E) was observed. (Philips 1.5 Tesla MR, Istanbul/Turkey. Godalinium contrast material $20 \mathrm{mg}$ was admitted).

factor for local recurrence of MLS is the surgical margins and the major factor for metastasis is the histological grade and the percentage of round cells of the tumor $[3,14]$.

In differantial diagnosis some tumors which contain myxoid tissue such as extraskeletal myxoid chondrosarco$\mathrm{ma}$, intramuscular myxoma, ganglion and myxoid malignant fibrous histiocytoma should be considered. Unlike MLS, these tumors tend to be homogeneous and have well defined contour [6].

\section{Conclusion}

Depends on the anatomical site, perineal location (the rare region) of the low attenuation in dansity or intensity cystic lesions, as was in our case, mixoid liposarcoma should be consider in differantial diagnosis. It should be kept in mind that it is of a great value to obtain the contrast enhanced imaging in order to distinguish the cystic lesions from the solid masses.

\section{Abbreviations}

MLS: Myxoid liposarcoma; CT: Computed Tomography; MRI: Magnetic Resonance Imaging; US: Ultrasonography; STIR: Short Tau Inversion Recovery. 


\section{References}

1. Enzinger FM, Weiss SW. Soft tissue tumors. 3rd ed. St Louis, Mo: Mosby-Year Book,1995; 431-466.

2. Benjaminov O, Gutman H, Nyabanda R, Keinan R, Sabach G, Levavi H. Myxoid liposarcoma: an unusual presentation. AJR Am J Roentgenol. 2007;188(3):817-821.

3. Loubignac F, Bourtoul C, Chapel F. Myxoid liposarcoma: a rare soft-tissue tumor with a misleading benign appearance. World J Surg Oncol. 2009;7:42.

4. Young B. Jeong, Ho S. Park, Dae W. Kim, Myung K. Kim, Jong K. Park, Hyung J. Kim, Young G. Kim. Intrascrotal Myxoid Liposarcoma of Perineal Origin. Curr Urol 2008;2:161-163.

5. Sung MS, Kang HS, Suh JS, Lee JH, Park JM, Kim JY, Lee HG. Myxoid liposarcoma: appearance at MR imaging with histologic correlation. Radiographics. 2000;20(4):1007-1019.

6. Peterson KK, Renfrew DL, Feddersen RM, Buckwalter JA, el-Khoury GY. Magnetic resonance imaging of myxoid containing tumors. Skeletal Radiol. 1991;20(4):245-250.

7. Evans HL. Liposarcomas and atypical lipomatous tumors: a study of 66 cases followed for a minimum of 10 years. Surg Pathol 1988; 1:41-54.

8. Kransdorf MJ. Malignant soft-tissue tumors in a large referral population: distribution of diagnoses by age, sex, and location. AJR Am J Roentgenol. 1995;164(1):129134.

9. Jelinek JS, Kransdorf MJ, Shmookler BM, Aboulafia AJ, Malawer MM. Liposarcoma of the extremities: MR and CT findings in the histologic subtypes. Radiology. 1993;186(2):455-459.

10. Ma LD, McCarthy EF, Bluemke DA, Frassica FJ. Differentiation of benign from malignant musculoskeletal lesions using MR imaging: pitfalls in MR evaluation of lesions with a cystic appearance. AJR Am J Roentgenol. 1998;170(5):1251-1258.

11. Kransdorf MJ, Murphey MD. The use of gadolinium in the MR evaluation of soft tissue tumors. Semin Ultrasound CT MR. 1997;18(4):251-268.

12. Beltran J, Chandnani V, McGhee RA, Jr., KursunogluBrahme S. Gadopentetate dimeglumine-enhanced MR imaging of the musculoskeletal system. AJR Am J Roentgenol. 1991;156(3):457-466.

13. Ramasamy P, Md Nor A, Kamil NA, Harun N, Yusof MM, Md Hassan MZ. Perineal myxoid liposarcomas: a case report and literature review. Malays J Med Sci. 2010;17(4):62-66.

14. Fiore M, Grosso F, Lo Vullo S, Pennacchioli E, Stacchiotti S, Ferrari A, Collini P, et al. Myxoid/round cell and pleomorphic liposarcomas: prognostic factors and survival in a series of patients treated at a single institution. Cancer. 2007;109(12):2522-2531. 